\title{
La négociation de signifi- cations professionnelles est-elle envisageable en formation? Propositions théoriques et illustrations
}

\section{Guillaume Escalié et Sébastien Chaliès}

Les principales propositions visant à aménager les dispositifs de formation professionnelle initiale des enseignants en vue de renforcer le partenariat université-école(s) sont fondées sur la théorie des communautés de pratique (Lave \& Wenger, 1991). Au sein de cette théorie, ces dispositifs sont envisagés comme des environnements ouverts de formation dans lesquels les significations accordées aux expériences de métier sont le résultat d'un processus de négociation. Or, les circonstances dans lesquelles se joue cette négociation restent assez obscures. Cet article vise à illustrer empiriquement des postulats issus d'une théorie de l'apprentissage (Nelson, 2008) et l'action collective (Wittgenstein, 1996) permettant de requalifier la négociation en formation professionnelle.

\section{Introduction}

Depuis plusieurs années, la recherche d'une amélioration de la formation professionnelle des enseignants est au cœur des politiques de l'Union européenne'. Parmi les propositions avancées par le parlement européen ${ }^{2}$, celle qui recommande de penser la formation des enseignants dans le cadre d'un véritable partenariat entre les universités et les écoles ${ }^{3}$ occupe une place de choix. S'inscrivant dans une volonté d'harmonisation des diplômes européens, la récente réforme de la formation des enseignants stagiaires (ES) engagée en France tend à répondre à cette orientation politique ${ }^{4}$.

Pour répondre à cette volonté, bon nombre d'universités ont essayé de redéfinir leur partenariat avec les écoles de sorte qu'il puisse concourir à la mise en œuvre d'une formation basée sur le principe d'une alternance davantage intégrative (Malglaive, 1994). Cette dernière se caractérise par une forte mise en cohérence des différentes périodes de l'alternance (formation universitaire d'un côté et expériences professionnelles de l'autre) et par une collaboration accrue entre l'ensemble de ses acteurs. Pour ce faire, des aménagements des dispositifs usuels de formation ont été mis en place. Ces derniers, fondés pour la plupart 
sur le modèle des communautés de pratique (Lave \& Wenger, 1991) actuellement privilégié au sein de la littérature scientifique, présentent trois principaux intérêts (Escalié \& Chaliès, 2011a). Le premier est relatif à la nature de l'engagement (Wenger, 1998) des différents acteurs, formateurs et formés, au sein des communautés de pratique. Un processus de négociation des significations à accorder aux expériences de métier est en effet rendu possible au sein de ces dernières (Sim, 2006), ce qui favorise la formation des ES mais aussi des conseillers pédagogiques (CP) dans les écoles et des formateurs de l'université (FU). Nommé imagination (Wenger, 1998), le deuxième intérêt est relatif aux possibilités offertes aux formateurs de mieux articuler les composantes théorique et pratique de la formation et d'atténuer ainsi le choc de la pratique aux ES (Korthagen, 2010). Nommé alignement (Wenger, 1998), le dernier intérêt est relatif au renforcement de la collaboration entre les différents acteurs de la communauté de pratique du fait de leur adhésion à un projet de formation partagé (Korthagen, Loughran \& Russell, 2006).

Le caractère privilégié alloué à ce modèle reste toutefois discutable, notamment si l'on s'attache à questionner ses soubassements théoriques. Bien que central, le postulat selon lequel les significations des expériences de métier sont nécessairement négociées par les membres de la communauté (formés et formateurs) n'est par exemple que partiellement étayé. Au cœur de ce modèle, la formation est en effet considérée comme le résultat de la participation des formés à des pratiques professionnelles constitutives de la communauté. Au cours de cette participation, sont engagées des négociations implicites et/ou explicites des significations attribuées aux expériences de métier rencontrées (Lave \& Wenger, 1991). Ces négociations de significations sont d'autant plus importantes qu'elles permettent théoriquement aux formés de passer d'une participation périphérique, comme pour tout nouveau venu (new comer), à une participation plus centrale et légitime caractérisant tout membre principal (old timer) (Alexander, Van Wyk, Bereng \& November, 2010) et, par voie de retour, de contribuer à la transformation de la communauté (Boylan, 2009). D’un point de vue théorique, les soubassements de ces négociations ne sont paradoxalement que peu développés. À la lecture des travaux relatifs à ce modèle, il est surtout possible de situer que ces négociations se font pour une large partie de façon implicite (Lave \& Wenger, 1991) par l'intermédiaire d'interactions non verbales et/ou d'alignements informels de la pratique des formés avec celle des autres membres (Rogoff, Matusov \& White, 1996) au cours de leur participation aux pratiques professionnelles constitutives de la communauté. Dans une moindre mesure, il semble aussi possible de considérer que ces négociations puissent se faire dans le cadre d'une participation des formés à des pratiques porteuses d'interactions verbales, explicites cette fois, avec les autres membres de la communauté professionnelle (Billett, 2009). Néanmoins, les circonstances dans lesquelles ces négociations explicites de significations se réalisent restent obscures. C'est à ce niveau que se situe l'objet de cet article. Par l'intermédiaire d'une explicitation puis d'une 
illustration des trois principaux postulats théoriques constitutifs d'une conceptualisation originale de la formation professionnelle, cet article cherche en effet à préciser les circonstances de formation au sein desquelles les négociations explicites de significations peuvent avoir lieu et aboutir à la formation des ES.

\section{Cadre théorique}

Cet article prend appui sur une conceptualisation de la formation professionnelle établie à partir de postulats empruntés à une théorie générale de l'apprentissage (Nelson, 2008) et de l'action collective (Wittgenstein, 1996). Compte tenu de l'objet de l'étude, seuls les trois principaux postulats de cette conceptualisation sont ci-après explicités. Ils permettent en effet de qualifier ce que pourrait être les circonstances d'une négociation explicite de significations en formation professionnelle.

Enseigner des règles aux ES pour leur permettre de signifier autrement leurs expériences professionnelles Le premier postulat est celui selon lequel la signification adéquate (au sens d'acceptée et de partagée par la communauté de formateurs) par les ES des expériences professionnelles vécues en classe suppose un apprentissage préalable de règles (Wittgenstein, 1996). L'apprentissage par les ES de ces règles nécessite l'engagement des formateurs, non pas dans une activité de négociation de significations, mais dans une activité d'enseignement ostensif (Wittgenstein, 1996) par laquelle ils fondent la signification des expériences professionnelles considérées comme exemplaires. Pour ce faire, ils dressent ostensivement pour chacune de ces expériences un lien de signification (Wittgenstein, 1996) entre, (i) l'expérience langagière visant à la nommer, (ii) les exemples décrits et/ou montrés en correspondance et (iii) les résultats qui y sont usuellement associés dans la communauté. Par exemple, lorsque les ES apprennent en début d'année scolaire ce que c'est que [«Prendre en main la classe»], ils n'apprennent pas seulement cet étiquetage langagier ; ils apprennent le lien entre cet étiquetage professionnel, les expériences exemplaires placées en correspondance par les formateurs (comme [«Faire l'appel dans le calme»] et [«Présenter succinctement l'objectif de la leçon aux élèves»]) et les résultats qui y sont associés (comme [«Mettre les élèves rapidement au travail»]). Ces liens de significations enseignés, et donc initialement non négociables, sont ensuite de véritables mètres étalons (Williams, 2002) lorsque les ES agissent dans de nouvelles situations professionnelles. Ces derniers s'appuient alors en effet sur ces échantillons d'expériences exemplaires ostensiblement montrés pour signifier les évènements rencontrés et essayer de s'y adapter. 


\section{Contrôler les premiers suivis de la règle par les ES}

Les formateurs s'engagent ensuite dans une activité d'accompagnement des premiers suivis par les ES des règles préalablement enseignées dans de nouvelles situations de formation et/ou de classe. Par cet accompagnement, ils visent à rendre possible mais aussi et surtout à faire en sorte que ces premiers suivis aboutissent aux résultats attendus y étant usuellement associés. Ce n'est en effet qu'au travers du constat de ces résultats attendus que les ES peuvent théoriquement associer consubstantiellement une intention professionnelle aux règles enseignées (Berducci, 2004; Ogien, 2007). Autrement dit, suivant correctement les règles préalablement enseignées et constatant les résultats qui leur sont usuellement associés dans la communauté professionnelle, les ES finalisent l'apprentissage de ces règles et peuvent alors se développer à partir de celles-ci (Nelson, 2008). Théoriquement, lors de ces premiers suivis, l'activité des formateurs ne peut toujours pas être assimilée à une activité de négociation de significations. Les formateurs contrôlent en effet leur conformité (Nelson, 2008) et s'engagent si nécessaire dans une activité d'explications ostensives (Wittgenstein, 1996). Ils s'efforcent alors de multiplier les exemples décrits et/ou montrés pouvant être associés aux règles enseignées de sorte de lever les incompréhensions ou mésinterprétations des ES et leur permettre, au final, de les suivre seuls et de façon acceptable, c'est-à-dire comme convenu dans la communauté professionnelle.

\section{Accompagner le développement des ES}

Lorsque les règles sont apprises, leurs suivis par les ES dans de nouvelles situations de classe et/ou de formation nécessitent leur interprétation, autrement dit leur négociation. Ces suivis renvoient en effet théoriquement à un usage extensif des liens de signification construits et appris, engageant les ES dans un développement professionnel. Ce développement s'effectue sur la base de l'identification par les ES d'un air de famille (Wittgenstein, 1996) entre les circonstances constitutives de la situation en cours et celles constitutives des situations dans lesquelles les règles ont été apprises. A partir des liens de signification appris, les ES ordonnent alors finalement un réseau complexe de ressemblances, interprètent au sens de comprennent la nouvelle situation en cours et in fine s'y adaptent. Ce postulat d'un développement d'instruments psychiques chez le sujet, conséquent à l'apprentissage de concepts nouveaux, n'est pas spécifique à cette approche et se retrouve aussi dans les recherches sur l'activité humaine en psychologie historico-culturelle (Vytgosky, 1978).

Ces situations de développement ne sont donc plus l'occasion pour les formateurs de contrôler le caractère adéquat ou inadéquat des significations associées par les ES aux évènements rencontrés. Elles leur permettent plutôt d'aider les ES à s'engager dans des suivis interprétés des règles apprises, c'est-à-dire de les aider à en négocier la signification pour mieux s'y adapter. Par exemple, en confrontant leurs propres styles d'enseignement avec ceux des formateurs, ils peuvent controverser avec eux sur la pertinence de tel ou tel nouvel exemple constitutif de la règle considérée. 


\section{Méthode}

Afin d'illustrer les postulats théoriques développés en amont, nous nous appuyons sur les résultats d'une étude présentée ci-après dans ses grandes lignes.

\section{Le dispositif de formation support à l'étude}

Le dispositif support à cette étude de cas longitudinale était celui de la Visite Formative en établissement scolaire mis en place lors de l'année de titularisation des ES, c'est-à-dire lors de la deuxième année d'IUFM ${ }^{5}$ consacrée à la formation professionnelle initiale au moment de l'étude. Il avait été initialement sélectionné car il était le seul dispositif nécessitant la mise en place d'un travail de type communauté de pratique entre les différents formateurs (CP et FU) et les formés. Réparties sur le deuxième trimestre de l'année scolaire, les trois visites se structuraient autour de deux temps successifs: un temps d'enseignement (une leçon) en responsabilité mené par un ES avec sa classe sous l'observation conjointe d'un $\mathrm{CP}$ et d'un FU, suivi d'un temps de formation collectif de type entretien de conseil pédagogique (ECP) post-leçon impliquant l'ensemble des acteurs de la triade.

\section{Recueil des données}

Des données d'enregistrement ont d'abord été recueillies pendant l'ECP de l'ensemble des visites. Une caméra vidéo, ainsi qu'un micro HF positionné entre les différents acteurs, ont été utilisés pour ce recueil.

Des données dites d'autoconfrontation ont ensuite été recueillies au cours d'enregistrements audio-vidéo. Tous les acteurs ont réalisé un entretien d'autoconfrontation (EAC) relatif à l'ECP. L'ES a aussi réalisé un EAC relatif à la leçon menée en amont de chaque ECP. Au total, treize EAC d'une durée d'une heure ont été réalisés. Les EAC ont été réalisées afin de recueillir le plus grand nombre d'éléments possibles pour reconstituer a posteriori les règles apprises et/ou suivies par les acteurs pour signifier leurs actions et/ou celles d'autrui visionnées. Pour ce faire, toutes les autoconfrontations ont été réalisées à partir du même protocole. Dans le détail, les questions posées par le chercheur ont visé à inciter l'acteur à: porter un jugement sur l'action visionnée (par exemple: «Qu'est-ce que tu penses de cette remarque ?») et la nommer (par exemple: "Quand tu dis: "ce n'est pas clair», qu'est-ce qui n'est pas clair ?»); justifier (au sens d'étayer) son jugement (par exemple: «Qu'est-ce qui te fait dire que le conseil que tu lui donnes n'est pas pertinent?»); décrire les résultats attendus ou constatés de l'action réalisée (par exemple: "Qu'est-ce que tu attends quand tu lui imposes ce principe sans même le négocier ?»). Les relances ont été effectuées soit par une demande de précision (par exemple: «Je ne comprends pas pourquoi tu considères cette action comme intéressante, peux-tu m'expliquer?»), soit par la mise en jeu d'une controverse plaçant l'acteur interviewé face à des contradictions apparentes (par exemple: «Tu me dis là que c'est plutôt bien de différencier alors que tu disais juste avant 
que c'est à cause de ton choix de différencier que tu as mis trop de temps pour présenter les situations aux élèves»).

\section{Traitement des données}

Le traitement a été réalisé afin d'identifier les règles apprises et/ou suivies par les acteurs lors des ECP. Pour ce faire, les données recueillies ont été traitées en trois étapes successives (Chaliès, Bertone, Flavier \& Durand, 2008):

(i) Après la retranscription verbatim de l'ensemble des données recueillies, le corpus a été découpé en unités d'interaction. Ces unités ont été délimitées à partir de l'objet des significations attribuées par les acteurs aux expériences de classe faisant l'objet d'une discussion entre l'ES et les formateurs lors de l'ECP.

(ii) Pour chaque unité d'interaction, l'étayage de la signification attribuée par les acteurs a été identifié. L'étayage a été associé à l'ensemble des circonstances évoquées par les acteurs pour expliquer au chercheur la façon de s'y prendre pour signifier l'expérience de classe objet de la discussion.

(iii) Pour chaque unité d'interaction, la règle suivie par l'acteur a été formalisée. Par convention, chaque règle a été étiquetée à partir (a) de l'objet de la signification attribuée par l'acteur, (b) de l'ensemble des circonstances évoquées par l'acteur pour étayer cette signification et (c) des résultats constatés et/ou attendus. Dans sa forme, chaque règle a été formalisée ainsi: [ fication» vaut pour "ensemble des circonstances évoquées pour étayer la signification» ce qui obtient comme résultat «ensemble des résultats constatés et/ou attendus»].

\section{Résultats}

La présentation des principaux résultats empiriques obtenus lors de l'étude présentée ci-dessus poursuit deux objectifs. Tout d'abord, elle vise à illustrer les trois principaux postulats théoriques constitutifs de la conceptualisation de la formation professionnelle développée. Complémentairement, elle cherche à renseigner les circonstances de formation au sein desquelles les négociations explicites de significations peuvent, ou ne peuvent pas, avoir lieu et aboutir à la formation des ES.

\section{L'absence de négociation lors de l'enseignement ostensif d'une nouvelle règle}

Ce premier résultat montre que lors de leur engagement dans une activité d'enseignement de nouvelles expériences professionnelles, les formateurs ne cherchent pas à en négocier leur signification. Ils fixent au contraire ostensivement ces dernières en dressant des liens de signification.

Lors de l'ECP relatif à la première visite formative (fin janvier), l'ES et les formateurs ont abordé un épisode de classe nommé [«la présentation des situa- 
tions aux élèves»]. Au cours de leurs EAC respectifs, chacun des acteurs s'est attardé avec le chercheur sur cet épisode. Ils se sont tous accordés pour juger que l'activité menée par l'ES à ce moment de la leçon manquait de clarté du fait notamment que deux situations différentes soient présentées simultanément aux élèves.

Il est à ce niveau intéressant de pointer que cette attribution d'une signification partagée à l'épisode de classe considéré peut être mise en lien avec l'activité des formateurs lors de l'ECP précédant leur EAC. Tel que l'illustre l'extrait suivant issu de cet ECP, le FU s'attache lors de ce temps de formation à enseigner à l'ES qu'il doit "faire des choix» lorsqu'il présente les situations aux élèves alors que pour le moment il semble vouloir «tout faire». Ceci est d'autant plus important que «c'est le métier» (Extrait 1).

\section{Extrait 1}

FU: Là on va aller sur un aspect où je pense que là-dessus il faut que tu évolues, c'est quand tu présentes la situation d'apprentissage. On revient juste sur cet aspect-là. Là, tu dois faire des choix, c'est le métier tu dois faire des choix. Parce qu'il y a un moment donné, tu es resté quatre minutes en monologue à expliquer sur le premier groupe. Et en fait, on a l'impression que tu veux tout faire.

Lors de son EAC, le FU s'est arrêté avec le chercheur sur ce moment de l'ECP où il enseigne la règle étiquetée ["présenter des situations aux élèves»] (Extrait 2).

\section{Extrait 2}

FU: Il (l'ES) a voulu tout faire. Sauf que présenter c'est faire des choix. Il y a des moments où l'on ne peut pas tout dire, où l'on ne peut pas tout faire et il faut faire des choix sur comment on place les choses, toujours dans un souci de l'élève.

$\mathrm{CH}$ (Chercheur): Tu enchaînes.

FU: Et puis c'est vrai que c'est clair au moins. Et nous (les formateurs), on est d'accord sur le fait que quand tu présentes, tu ne passes pas vingt minutes à expliquer. Les élèves ne vont pas attendre vingt minutes qu'on leur donne les consignes. Donc là-dessus, ce n'est pas négociable.

A cet instant de l'EAC, le FU suit la règle [«Présenter les situations» vaut pour «faire des choix» ce qui obtient comme résultats de "ne pas passer vingt minutes à expliquer une situation» et "ne pas faire attendre les élèves»]. Bien qu'il ne soit pas parvenu à l'enseigner de façon ostensive à l'ES («Là, tu dois faire des choix, c'est le métier tu dois faire des choix. Parce qu'il y a un moment donné, tu es resté quatre minutes en monologue à expliquer sur le premier groupe»), le FU insiste toutefois lors de son EAC sur le caractère non négociable de cette signification au sein de la communauté professionnelle («ce n’est pas négociable»). 
Parallèlement, lors de son propre EAC relatif à ce temps de formation, l'ES souligne que le fait que le FU ait été «directif» n'a pas été complètement étranger à sa façon de signifier son activité de présentation des situations aux élèves (Extrait 3).

\section{Extrait 3}

$\mathrm{CH}$ : Et C. (le $F U$ ), comment tu le perçois là dans cette relation?

ES: Déjà là, je l'ai senti..., directif. J'ai senti que, il dit... «On était d'accord entre nous», il y a souvent ces injonctions là: "Ça, ce n'est pas bien ce que tu as fait là, il y a un truc qui n'est pas bon». Ok, bon, d'accord. Il dit: «Bon on voit que tu as essayé de faire quelque chose et maintenant on peut réfléchir ensemble».

A cet instant de l'EAC, l'ES précise que le FU ponctue souvent ses propos par des «injonctions» telle que "ce n'est pas bien ce que tu as fait là». Ces injonctions ne lui donnent pas d'autres choix que de s'engager dans une démarche réflexive collective («maintenant on peut réfléchir ensemble») pour parvenir à se mettre d'accord sur les significations à attribuer aux évènements de la classe et éventuellement sur les actions à mener pour s'y adapter.

Au final, ce résultat montre que l'ES et le FU parviennent à s'accorder sur la signification donnée à l'épisode relatif à la présentation des situations aux élèves. Il montre plus précisément que cet accord de signification n'est pas le résultat d'une négociation explicite mais plutôt de l'engagement du formateur dans un enseignement qui, même s'il reste encore peu ostensif, n'en est pas moins prescriptif et non négociable du point de vue du métier.

\section{Les explications ostensives pour faire apprendre des significations non négociables}

Ce deuxième résultat montre que suite à l'enseignement de nouvelles expériences professionnelles considérées comme exemplaires dans la communauté, les formateurs mènent une activité d'explication ostensive afin de lever d'éventuelles mésinterprétations ou incompréhensions chez les ES. L'accord qui est alors recherché avec ces derniers ne relève toujours pas d'une négociation explicite de significations.

A l'occasion de l'ECP relatif à la deuxième visite (mi-février), les formateurs et l'ES ont une nouvelle fois discuté de son activité de présentation des situations aux élèves. Lors de son EAC, le FU précise au chercheur qu'il s'attarde sur cet épisode de classe car les premiers suivis par l'ES de la règle qui lui a été enseignée sont peu conformes à ses attentes et donnent à penser qu'une mésinterprétation ait pu avoir lieu lors de son enseignement. Il relève, plus précisément, qu'un des éléments d'étayage de cette règle est celui relatif à la nécessité qu'il n'y ait qu' "un seul temps", autrement dit qu'une seule situation soit présentée à la fois. Or, lors de sa leçon, l'ES propose encore «deux situations» dans le même temps de présentation. Il ne répond dons toujours pas à "ce que les formateurs attendent» (Extrait 4). 


\section{Extrait 4}

$\mathrm{CH}$ : Là, tu crois qu'il (l'ES) résiste?

FU: Si on lui demande d'expliquer ce que sont ses situations, c'est que ce n'était pas clair et que l'on était obligé d'y prendre un temps. Donc là je me dis: «Bon voilà, il va nous démontrer ses différents temps». Comment après on arrive au fait que bon, simplement il y a deux situations et ce n'est pas ce que l'on (les formateurs) attend et comment on fait?

$\mathrm{CH}$ : C'est-à-dire, je ne comprends pas pourquoi vous n'êtes pas un peu plus sur: «Non mais attends, que ce soit deux temps différents ou pas, la présentation...».

FU: Là, j'aurais dû dire: «Au-delà de la première et de la deuxième situation, il faut un seul temps; si tu présentes deux temps, ils (les élèves) s’y perdent».

Il est intéressant de souligner que, du point de vue du FU, l'ES ne suit pas la règle qui lui a été préalablement enseignée. En classe, l'ES semble en effet suivre la règle [«Présenter les situations» vaut pour "présenter séparément la première situation et la deuxième situation»] alors même que lui a été enseignée une autre règle ([«Présenter les situations» vaut pour "présenter un seul temps à la fois» ce qui obtient comme résultat de «ne pas perdre les élèves»]). Constatant ce malentendu, le FU ne cherche pas à négocier la signification considérée. Il s'engage avec l'aide de la CP dans des explications afin de lever la mésinterprétation de l'ES et d'aboutir à un accord quant à la façon attendue dans la communauté de présenter des situations aux élèves. L'extrait d'ECP suivant renseigne cette activité d'explication (Extrait 5).

\section{Extrait 5}

FU: Tu as voulu donner encore deux situations au même moment.

ES: Je n'ai pas voulu donner deux situations, j'ai voulu donner une situation et son évolution.

FU: Comme la dernière fois.

CP: Et ouais.

ES: Non, la dernière fois, j'ai donné deux situations vraiment.

FU: Mais c'est comme la dernière fois. Tu as deux temps que tu veux donner en une fois aux élèves.

CP: Ouais. C'est l'histoire des deux temps.

ES: Oui, je comprends.

FU: Pour faire le bilan, on est d'accord que quand tu enseignes, tu ne peux pas présenter aux élèves deux choses en même temps.

CP: Deux choses en même temps.

ES: Oui.

Cet extrait illustre l'engagement des formateurs dans une activité d'explication ostensive d'une règle préalablement enseignée. Plus précisément, il permet de 
montrer que les formateurs cherchent à construire un accord avec l'ES quant au fait qu'il n'est pas possible de "présenter aux élèves deux choses en même temps". Pour ce faire, le FU renseigne un nouvel élément d'étayage de la règle relatif au nombre de temps à communiquer aux élèves dans un même temps de parole («on est d'accord que quand tu enseignes, tu ne peux pas présenter aux élèves deux choses en même temps»), indépendamment de la nature de ces temps (une situation et son évolution ou deux situations différentes). Le FU associe donc de nouvelles circonstances exemplaires à la règle enseignée en vue de dissiper le malentendu avec l'ES et de l'engager dans des premiers suivis de cette règle conformes.

Lorsqu'il est autoconfronté sur cet extrait d'ECP, l'ES rend aussi compte du caractère «non négociable» de la signification à attribuer à la présentation des situations (Extrait 6).

\section{Extrait 6}

$\mathrm{CH}$ : Qu'est-ce qu'il (le FU) fait là?

ES: Il dit: «Bon, on fait le bilan; tu es bien d'accord avec ça?». Je pense qu'il s'assure qu'il n'y ait pas de malentendu sur tout ce que l'on a dit.

$\mathrm{CH}$ : Qu'il n'y ait pas de malentendu, ça veut dire quoi?

ES: Malentendu par rapport à ce qu'il veut me faire passer. À mon avis, il a un truc non négociable à me dire, c'est comme ça et ce n'est pas autrement.

$\mathrm{CH}$ : Ouais.

ES: Le «On est bien d'accord», si je lui dis que je ne suis pas d'accord, il va me dire: «De toute façon...». Je m'attends à ce qu'il me dise: "Ce n'est pas négociable».

A cet instant de l'EAC, l'ES précise que par son bilan le FU insiste sur le caractère «non négociable» de ce qui est enseigné. Le FU cherche selon lui à s'assurer "qu'il n'y ait pas de malentendu» quant à la signification de la règle abordée relative à la présentation des situations aux élèves. Notons pour conclure que, si l'explication ostensive du FU permet d'aboutir à un accord avec l'ES quant à la façon de signifier la présentation des situations, l'apprentissage de cette règle ne sera finalisé qu'une fois que la règle aura été suivie de façon conforme en classe et que les résultats qui y sont usuellement associés auront pu être effectivement constatés. L'ES pourra alors s'engager dans des suivis interprétés de la règle considérée, c'est-à-dire s'engager dans sa négociation compte tenu de la singularité des situations de classe rencontrées.

\section{La négociation de significations comme ressource du développement professionnel}

Ce dernier résultat montre que l'engagement de l'ES et de ses formateurs dans la négociation de significations peut avoir lieu suite à l'apprentissage. Il est alors source de développement professionnel.

A l'occasion de l'ECP relatif à la troisième visite formative (mi mars), l'ES et les formateurs ont été amenés à discuter d'un épisode de classe nommé [«l'échauf- 
fement des élèves»]. Dans l'extrait suivant issu de son EAC, l'ES précise qu'il a cherché à s'organiser pour ne plus simplement faire un échauffement parce qu'il fallait le faire lors de la venue des formateurs, mais davantage pour que ça serve ensuite aux élèves lors de la leçon. Il juge donc avoir modifié son échauffement pour qu'il prépare les élèves à la leçon («je cherche à faire un échauffement qui sert à») et plus seulement pour «faire plaisir» aux formateurs comme cela était le cas lors des visites précédentes (Extrait 7).

\section{Extrait 7}

$\mathrm{CH}$ : J'ai l'impression que tu as basculé dans ta conception.

ES: Oui, parce qu'ils (les formateurs) m'ont fait évoluer. A force de me dire «tu fais un échauffement parce qu'il faut faire un échauffement»... Et puis, c'est ce que je te disais, avant, je faisais un échauffement pour leur faire plaisir. Là, le fait de me faire réfléchir là-dessus et de vraiment travailler là-dessus, je cherche à faire un échauffement qui sert aux élèves compte tenu de l'activité qu'ils vont pratiquer.

CH: D'accord.

ES: Et donc, du coup, plus à n’importe quel prix.

A cet instant de l'EAC, l'ES suit la règle ["Echauffer les élèves» vaut pour "proposer des exercices utiles compte tenu de l'activité pratiquée»]. Le suivi de cette règle témoigne de son intention de proposer aux élèves un échauffement leur permettant d'en tirer des bénéfices pour la suite de la leçon. Dans l'extrait d'EAC suivant, l'ES précise qu'il a choisi de mettre en œuvre un échauffement fondé sur la méthode de la Proprioception Neuro-Facilitante ${ }^{6}$ (PNF) car il juge qu' elle lui permet de répondre à ce que lui ont préalablement enseigné les formateurs. En ce sens, cette méthode lui paraît «utile» (Extrait 8).

\section{Extrait 8}

ES: L'échauffement, c'est six secondes d'étirement non maximal, six secondes de contraction et six secondes de dynamique. Et elle, ce qu'elle (la CP) fait, c'est six secondes d'étirement, six secondes de contraction et après elle fait faire une longueur de montées de genoux ou de talons-fesses aux élèves.

$\mathrm{CH}$ : Oui.

ES: Moi, si j'utilise cet échauffement, ce n'est pas juste pour échauffer, c'est parce qu'il est utile.

$\mathrm{CH}$ : C'est que tu prêtes une importance à l'échauffement au sens d'échauffer le corps.

ES: Oui, mais elle (la CP), elle se moque de dénaturer cet échauffement tant qu'il est spécifique à sa course. Et du coup, est-ce qu'il échauffe réellement de la même façon que celle dans laquelle il a été pensé?

$\mathrm{CH}$ : D'accord.

ES: Et ça me gêne. 
L'ES suit la règle [«L'échauffement en PNF est utile et échauffe réellement» vaut pour «la méthode de la PNF ne doit pas être dénaturée et doit être réalisée de la même façon qu'elle a été pensée»]. Le suivi de cette règle marque un développement professionnel de la part de l'ES. En effet, à travers l'utilisation de la méthode de la PNF, l'ES cherche à faire usage dans de nouvelles circonstances de la règle apprise antérieurement, toujours dans l'intention de rendre l'échauffement utile et spécifique comme le lui ont enseigné les formateurs.

Alors que l'ES juge avoir pris en considération les éléments des séquences de formation antérieures pour faire évoluer sa façon d'échauffer ses élèves, la CP n'est toujours pas convaincue par la pertinence de sa façon de faire. Dans l'extrait d'EAC suivant, elle juge qu'il n'a pas encore proposé d'échauffement qui prépare la suite de la leçon puisqu' «il n'est pas rentré dans les fondamentaux» de l'athlétisme, activité support de la leçon (Extrait 9).

\section{Extrait 9}

CP: J'essaye de lui (l'ES) faire prendre conscience qu'on rentre dans une activité athlétique avec des contenus athlétiques.

$\mathrm{CH}$ : Donc là, concrètement, l'idée, c'est quoi?

CP: L'idée, c'est qu'il (l'ES) me dise en quoi son échauffement va préparer à la suite.

$\mathrm{CH}$ : Donc, il n'a pas répondu pour toi.

CP: Non, pas assez. Parce qu'il n'est pas rentré dans les fondamentaux de l'athlétisme.

$\mathrm{CH}$ : D'accord. Donc, tu es insatisfaite de ce qu'il (l'ES) t'a répondu.

CP: C'est-à-dire que dans sa discussion, il pense avoir répondu à ce que j'avais demandé, de faire une liaison de son échauffement avec l'activité pratiquée mais moi je ne suis pas sûr que ce soit un échauffement d'athlétisme.

Dans cet extrait, la CP suit la règle [«Faire un échauffement qui prépare la suite» vaut pour «rentrer dans les fondamentaux de l'activité» ce qui obtient comme résultat de "rentrer dans une activité athlétique avec des contenus athlétiques»]. Par ce suivi, elle juge que malgré les apports faits lors de la séquence de formation précédente, l'ES ne fait toujours pas faire aux élèves un «échauffement spécifique» qui prépare la leçon, crest-à-dire qui établisse une «liaison entre l'échauffement et l'activité pratiquée». Alors que l'ES juge suivre conformément la règle apprise lors de la séquence de formation antérieure, la CP considère donc sur la base de ce qu'elle observe que ce n'est pas le cas. Ce désaccord engage alors l'ES dans une activité de négociation de significations au sujet de la règle apprise. Dans l'extrait d'EAC suivant, l'ES précise les raisons de ce désaccord. Il s'est efforcé d'utiliser la méthode de la PNF pour répondre aux attentes de formateurs en termes de mise en œuvre d'un échauffement utile aux élèves. Après tous les efforts qu'il a entrepris pour proposer un échauffement conforme selon lui aux attentes des formateurs, l'ES ne peut accepter le fait de réaménager une nouvelle fois sa façon de faire «en prenant des morceaux de tout et n'importe quoi» (Extrait 10). 


\section{Extrait 10}

ES: C'est peut-être la façon dont on me l'a appris à l'université, mais le but de cet échauffement, c'est d'échauffer sans fatiguer. Et donc il n'y a pas de distances, il n'y a pas de parcours et il n'y a pas de déplacements.

$\mathrm{CH}$ : Oui.

ES: Je pense qu'elle (la CP) cherche, c'est de rendre mon échauffement encore plus spécifique. Elle ne va pas plus loin. Moi, je pense que travailler à rendre un échauffement plus spécifique oui, mais pas pour finalement prendre des morceaux de tout et de n'importe quoi et faire un mix (mélange) dont je ne connais pas les réels effets.

Autrement dit, pour interpréter la règle, l'ES s'est appuyé sur des éléments de formation non enseignés par les formateurs mais qu'il a pu se procurer en se référant aux enseignements théoriques reçus à l'université à propos de la méthode de la PNF. Toutefois, ces éléments d'étayage s'avèrent différents de ceux mobilisés par la CP. L'ES est donc en désaccord avec la CP quant à la nature de son interprétation de la règle apprise. Il s'engage donc dans une négociation avec la CP. Dans l'extrait d'EAC suivant, l'ES complète ses propos en indiquant qu'il est prêt à accepter un autre exemple d'échauffement pour rendre son échauffement plus spécifique à la condition qu'on lui «montre que c'est efficace» (Extrait 11).

\section{Extrait 11}

$\mathrm{CH}$ : Qu'est-ce qu'elle (la $\mathrm{CP}$ ) te dit là?

ES: Elle me dit: «Bon, tu es attaché à un modèle mais il y a en d'autres qui sont peut-être efficaces». Et si on me montre qu'ils sont aussi efficaces, pourquoi pas...

$\mathrm{CH}$ : Là, finalement, elle ne te convainc pas simplement sur le fait de t'en parler.

ES: Non.

$\mathrm{CH}$ : Ça veut dire quoi si on me montre que c'est efficace?

ES: Moi, tel qu'on me l'a enseigné, il est efficace comme ça. Donc, si elle me montre qu'on peut avoir cette distorsion sans que l'on perdre les bénéfices de l'échauffement, pourquoi pas.

Dans cet extrait, l'ES suit la règle [«Accepter d'autres modèles» vaut pour «lui montrer qu'ils sont aussi efficaces» ce qui obtient comme résultat que «ne pas être convaincu sur le simple fait d'en parler»]. Pour être accompagné dans son développement, l'ES a donc semble-t-il besoin que la CP lui montre qu' «on peut avoir cette distorsion sans que l'on perdre les bénéfices de l'échauffement». Autrement dit, l'ES peut donc s'engager dans une négociation de significations avec les formateurs à la condition que ceux-ci soient en capacité de le convaincre, c'est-à-dire en capacité de l'amener à changer ses façons de faire en classe et de lui en faire constater le caractère professionnellement pertinent. 


\section{Discussion}

Cette étude de cas, en apportant une compréhension singulière du fonctionnement d'une triade $\mathrm{ES} / \mathrm{CP} / \mathrm{FU}$, vise à valider certains postulats théoriques permettant ensuite d'aménager de nouvelles situations de formation pouvant alors être généralisées. Autrement dit, les résultats saillants issus de cette étude qualitative peuvent servir à alimenter la démarche itérative présidant à la construction de dispositifs de formation et consistant à utiliser des résultats de recherche comme ressources pour optimiser voire diffuser et généraliser certaines pratiques de formation.

Les résultats permettent tout d'abord de souligner que, bien qu'ils soient pensés puis agencés selon le modèle des communautés de pratique, bon nombre de dispositifs usuels de formation ne constituent pas des espaces de négociations explicites de significations. Ils constituent plutôt des espaces de prescriptions de nouvelles significations des formateurs aux formés. Lorsque l'on s'attache à identifier les modifications effectives de la pratique professionnelle de classe des ES au cours de ces dispositifs, on peut effectivement repérer qu'elles sont toujours précédées d'une activité de formation lors de laquelle les significations ont été données à apprendre sans être négociées.

Dans le détail, les résultats de cette étude ont permis d'illustrer le postulat selon lequel pour toute nouvelle signification, les formateurs s'engagent dans une activité d'enseignement de règles professionnelles au cours de laquelle ils dressent de façon ostensive et non négociée des liens de signification (point 4.1. des résultats). Cette activité d'enseignement permet aux ES de signifier autrement (c'est-à-dire de façon adéquate dans la communauté) leur pratique de classe. Ce postulat semble d'ailleurs pouvoir être aussi étayé par les résultats de l'étude menée par Cothran et al. (2008), montrant que les ES préferrent «un style d'accompagnement directif» en comparaison avec une approche indirecte basée sur le modèle du "questionnement réflexif». Autrement dit, ce postulat amène à se distancier d'une position interactionniste radicale, dans laquelle la signification est envisagée comme étant continuellement renégociée dans des processus interactifs de participation. En envisageant la négociation de significations comme étant fondée sur une activité d'enseignement ostensif des formateurs, il invite à considérer qu'il ne suffit pas à un ES de «tremper» (Mottier-Lopez \& Allal, 2004) dans la communauté professionnelle pour en devenir un membre à part entière. Complémentairement, ce postulat invite aussi à discuter de la pertinence des dispositifs de formation qui ne seraient que le lieu d'une pratique réflexive jamais associée à l'enseignement de nouvelles règles professionnelles.

Par ailleurs, les résultats de l'étude ont permis d'illustrer le postulat selon lequel l'engagement des formateurs dans une activité d'enseignement ne peut suffire à l'apprentissage de toute nouvelle règle par les ES. Il apparaît en effet nécessaire que les formateurs accompagnent ces derniers lors de leurs premiers suivis, notamment en situation de classe, des règles préalablement enseignées 
(point 4.2. des résultats). C'est effectivement par cette activité d'accompagnement que les formateurs se donnent l'occasion de juger de l'adéquation des suivis des règles par les $\mathrm{ES}$ et, si nécessaire, de s'engager dans une activité complémentaire d'explication ostensive. Ils multiplient alors, sous forme d'exemples montrés et/ou décrits, les circonstances pouvant être associées aux règles préalablement enseignées afin de progressivement lever les incompréhensions et/ou les malentendus des ES. A ce niveau, c'est aussi le constat d'une non négociation des significations par les formateurs auprès des ES. Les formateurs doivent aussi en effet se donner les moyens de contrôler les premiers suivis des règles par les ES pour éventuellement les rendre adéquats aux attendus dans la communauté professionnelle considérée. A ce titre, la complémentarité établie entre les FU et les CP nécessite d'être davantage pensée pour que sa mise en œuvre aboutisse à de véritables collectif de formateurs (Escalié \& Chaliès, 2011b). Nos résultats suggèrent par exemple que les énoncés de règles génériques par les FU nécessitent une activité d'illustration et d'ancrage par et sur des échantillons de leçon des ES par les CP afin qu'ils en comprennent la signification et parviennent à construire leur propre style d'enseignement. D'un côté, les FU sont confrontés, contrairement aux $\mathrm{CP}$, à des collectifs d'ES en formation. De ce fait, ils sont contraints de fixer leurs apports à un niveau générique pour que tous les ES puissent en faire usage. L'activité d'enseignement des règles semble donc plutôt de leur ressort. D'un autre côté, compte tenu de leur expérience et de leur connaissance des circonstances d'enseignement des ES (par exemple en termes de possibilités et/ou de contraintes locales propres à l'établissement), les $\mathrm{CP}$ semblent a priori les mieux placés pour accompagner au quotidien les ES dans leurs suivis des règles préalablement enseignées et/ou apprises.

Enfin, les résultats de cette étude permettent d'illustrer le postulat selon lequel une négociation de significations peut se faire suite à l'apprentissage par les ES de nouvelles règles. Ayant appris ces dernières, les ES peuvent effectivement s'engager dans une activité d'interprétation de type "compréhension de la règle» (Ogien, 2007) avec l'aide des formateurs (point 4.3. des résultats). Pour autoriser cette négociation, il s'agit tout d'abord pour les formateurs de donner à entendre, à voir et/ou à vivre (en situation de formation et/ou de travail) aux ES le caractère inadapté de leurs suivis des règles considérées compte tenu de la singularité de la situation de travail. Par ce jugement portant sur l'inadéquation de ce qui est fait (notamment en termes des résultats obtenus), ils cherchent en quelque sorte à les convaincre de la nécessité de s'engager dans ce travail de formation complémentaire de type interprétation des règles apprises. Par la suite, le travail consiste à exemplariser de façon singulière la règle compte tenu des circonstances de suivi à venir. Paradoxalement, le fait que la négociation ouvre aux ES la possibilité de controverser avec les formateurs tend à rendre l'activité de ces derniers plus difficile. A ce niveau, l'accompagnement au développement proposé par les formateurs aux ES peut être considéré, à l'instar des travaux de Ria, Serres et Leblanc (2010) comme une ressource pour la formation à 
la réflexivité. Les controverses professionnelles entre les ES et les formateurs relatives au comment rendre possibles des usages extensifs des règles préalablement apprises apparaissent en effet comme une source d'approfondissement de la formation de donc de leur réflexion.

\section{Conclusion}

Cet article cherchait à développer et à illustrer une lecture théorique permettant de défendre le point de vue selon lequel les significations constitutives d'une communauté professionnelle ne sont pas a priori négociables en formation. Au final, il ne remet pas en question l'idée d'une possible négociation explicite des significations. Par contre, il spécifie les circonstances dans lesquelles cette négociation semble envisageable, ce qui représente une avancée par rapport au modèle des communautés de pratique.

Complémentairement, cette étude permet d'étayer le postulat d'une possible réflexion des formés afin qu'ils puissent négocier les significations qui leur ont été antérieurement apprises. Plus exactement, elle permet de poser le postulat selon lequel la réflexivité (en termes de compréhension) peut être assimilés à une activité de négociation de significations source de développement professionnel.

Il est finalement important de souligner que cet article n'interroge que ce que nous avons initialement défini comme la négociation explicite des significations. La négociation implicite, relevant d'une participation informelle des ES aux pratiques de la communauté, n'est ici pas abordée. Pour autant, on peut supposer qu'une large part de la formation relève de ce type de négociation notamment depuis que celle-ci se fait principalement en établissement scolaire. C'est certainement là une zone potentielle de recherche pour les prochaines années tant d'un point de vue théorique que méthodologique.

\section{Notes}

1 Journal Officiel de l'Union Européenne C119 (2009). Conclusions du Conseil Européen concernant un cadre stratégique pou la coopération européenne dans le domaine de l'éducation et de la formation.

2 Communication au Parlement européen (2007). Améliorer la qualité des études et de la formation des enseignants.

3 Le terme «écoles» vaut pour tous les établissements scolaires des premier et second degrés.

4 France: Ministère de l'enseignement supérieur et de la recherche (2010). Dispositif d'accueil, d'accompagnement et de formation des enseignants stagiaires des premier et second degrés et des personnels d'éducation stagiaire. Circulaire n 2010-037 du 25 Février 2010.

5 Lors de cette année de titularisation, la formation des ES était organisée sur un principe d'alternance entre des stages professionnels dans les écoles (à mi-temps) et des temps de formation à l'IUFM (à mi-temps). Cette organisation de la formation a été grandement réformée en 2010.

6 La PNF est une méthode d'échauffement qui présente l'originalité d'échauffer les muscles sans les fatiguer. L'échauffement de chaque muscle s'effectue sur place et passe par trois 
étapes successives durant chacune six secondes: une contraction non maximale du muscle, un étirement non maximal et une mobilisation dynamique sur place.

\section{Bibliographie}

Alexander, G., Van Wyk, M., Bereng, T. \& November, I. (2010). Legitimate peripheral participation (LPP): The case for recognition of prior learning sites and knowledge in South Africa's transforming education system. Teaching and Teacher Education, 26, (1), 45-52.

Berducci, D. (2004). Developmental Continuum Vygotsky through Wittgenstein: A New Perspective on Vygotsky's. Theory Psychology, 14, (3), 329-353.

Billett, S. (2009). Modalités de participation au travail: la dualité constitutive de l'apprentissage par le travail. In M. Durand \& L. Fillietaz (Éd.), Travail et formation des adultes (pp. 37-63). Paris: PUF.

Boylan, M. (2009). Ecologies of participation in school classrooms. Teaching and Teacher Education, 25, (1), 1-10.

Chaliès, S., Bertone, S., Flavier, E. \& Durand, M. (2008). Effects of collaborative mentoring on the articulation of training and classroom situations: A case study in the French school system. Teaching and Teacher Education, 24, (3), 550-563.

Cothran, D. , McCaughtry, N., Smigell, S., Garn, A., Kulinna, P., Martin, J. J. \& Faust, R. (2008). Teachers' preferences on the qualities and roles of a mentor teacher. Journal of Teaching in Physical Education, 27, 241-51.

Escalié, G. \& Chaliès, S. (2011a). Vers un usage européen du modèle des communautés de pratique en formation des enseignants. Revue Française de Pédagogie, 174, 107-118.

Escalié, G. \& Chaliès, S. (2011b). Apprendre des règles de métier: le rôle du collectif de formateurs. Recherche et Formation, 67, 149-164.

Korthagen, F., Loughran, J. \& Russell, T. (2006). Developing fundamental principles for teacher education programs and practices. Teaching and Teacher Education, 22, (8), 1020-1041.

Korthagen, F. (2010). Situated learning theory and the pedagogy of teacher education: Towards an integrative view of teacher behavior and teacher learning. Teacher and Teaching Education, 26, (1), 98-106.

Lave, J. \& Wenger, E. (1991). Situated learning: Legitimate peripheral participation. Cambridge: Cambridge University Press.

Malglaive, A. (1994). Alternance et compétences. Cahiers pédagogiques, 320, 26-28.

Mottier-Lopez, L. \& Allal, L. (2004). Participer à des pratiques d'une communauté classe: un processus de construction de significations socialement reconnues et partagées. In R. Richenmann \& C. Moro (Éd.), Significations et situations éducatives (pp. 59-84). Bruxelles: De Boeck.

Nelson, K. (2008). Wittgenstein and contemporary theories of word learning. New Ideas in Psychology, 4, (3), 1-13.

Ogien, A. (2007). Les formes sociales de la pensée. La sociologie après Wittgenstein. Paris: Armand Colin.

Ria, L., Serres, G. \& Leblanc, S. (2010). De l'observation vidéo à l'observation in situ du travail enseignant en milieu difficile: étude des effets sur des professeurs stagiaires. Revue Suisse des Sciences de l'Education, 32, (1), 105-120.

Rogoff, B., Matusov, E. \& White, C. (1996). Models of teaching and learning: participation in a community of learners. In Handbook of Education and Human Development (pp. 389-414). Oxford: Blackwell.

Sim, C. (2006). Preparing the professional experiences incorporating preservice teachers as communities of practice. Teaching and Teacher Education, 22, (1), 77-83.

Vygotsky, L. (1978). Mind in society: The development of higher psychological processes. Cambridge, MA: Harvard University Press. 
Wenger, E. (1998). Communities of practice. Cambridge: Cambridge University Press.

Williams, M. (2002). Tout est-il interprétation? In C. Chauviré \& R. Ogien (Éd.), La régularité (pp. 207-233). Paris: EHESS.

Wittgenstein, L. (1996). Recherches Philosophiques. Paris: Gallimard.

Mots-clés: Partenariat université-école(s), formation professionnelle, règles, apprentissage, négociation

\section{Wird die Verhandlung der Bedeutung von professionellen Erfahrungen in der Ausbildung sichtbar? Theoretische Vorschläge und Veranschaulichungen}

\section{Zusammenfassung}

Wesentliche Vorschläge zu einer verstärkten Zusammenarbeit von Universität und Schule/n im Rahmen der Grundausbildung von Lehrpersonen basieren auf der Theorie der Praxiseinheiten/Praxisgemeinschaften (Lave \& Wenger, 1991). Im Kern dieser Theorie werden sie als offene Ausbildungsumgebungen gedacht, in denen die der Berufserfahrung beigemessenen Bedeutungen das Ergebnis eines Verhandlungsprozesses sind. Die Bedingungen, unter denen dieser Prozess des Verhandelns abläuft, bleiben jedoch unklar.

Der vorliegende Artikel hat zum Ziel, die theoretischen Postulate, die aus einer Lerntheorie (Nelson, 2008) und einer Theorie des kollektiven Handelns (Wittgenstein, 1996) hervorgegangen sind, empirisch $\mathrm{zu}$ veranschaulichen. Dadurch wird eine Neubewertung der (Rolle von) Verhandlungen im Rahmen der beruflichen Ausbildung ermöglicht.

Schlagworte: Partnerschaft Universität-Schule, Grundausbildung, Berufsbildung, Verhandlung

\section{È possibile negoziare i significati professionali nella formazione? Proposte teoriche ed esempi}

\section{Riassunto}

La principale proposta per migliorare i programmi di formazione professionale degli insegnati al fine di rafforzare la sinergia tra formazione terziaria e mondo della scuola è quella basata sulla teoria delle Comunità di Pratica (Lave \& Wenger, 1991). In questa teoria, i programmi sono concepiti come ambienti aperti di apprendimento, nei quali i significati delle esperienza sono il risultato di un processo di negoziazione. Tuttavia, le circostanze in cui avviene tale negoziazione restano piuttosto indefinite. Questo articolo mira a illustrare empirica- 
mente alcuni postulati teorici che emergono da una teoria dell'apprendimento (Nelson, 2008) e dell'azione collettiva (Wittgenstein, 1996) per ri-qualificare la negoziazione nella formazione professionale.

Parole chiave: Sinergia scuola-università, formazione di base, formazione professionale, negoziazione

\section{Is the negotiation of professional meanings possible in training? Theoretical proposals and illustrations}

\section{Summary}

The main proposals to fit out the teachers' professional training programs in order to strengthen the university - school(s) partnerships are based on the theory of communities of practice (Lave \& Wenger, 1991). Within this theory, these programs are considered as open training environments in which the meanings of ongoing professional experiences result from a negotiation process. Yet, the circumstances in which takes place this negotiation remain rather dark. This article aims at illustrating empirically theoretical postulates stemming from a theory of learning (Nelson, 2008) and collective action (Wittgenstein, 1996) to re-qualify the negotiation in professional training.

Keywords: University-school partnership, vocation training, professional training, learning, negotiation 
